\title{
The role of apparent distance in the Poggendorff illusion
}

\author{
ALEXANDER E. WILSON and ALEXANDER W. PRESSEY \\ University of Manitoba, Winnipeg, Manitoba, Canada R3T 2N2
}

\begin{abstract}
This study was designed to test the hypothesis that apparent shrinkage of the distance between the oblique lines is responsible for the Poggendorff illusion. The results from one experiment, which provided an indirect test by increasing the length of the oblique arm, supported the shrinkage hypothesis. However, a second experiment, in which apparent distance was measured directly, did not support the hypothesis. Instead, the distance between the oblique lines appeared longer than a control distance. It was concluded that the argument, made by assimilation theory, that the Poggendorff illusion is caused by changes in the apparent distance between oblique lines must be reassessed.
\end{abstract}

If two portions of a single transverse line are interrupted by two separate parallel lines, the two portions do not appear collinear. Such a configuration is known as the Poggendorff illusion and is illustrated in Figure 1. In this case, the oblique line on the left appears to point above the oblique line on the right.

The oldest, most simple, and most popular explanation of this illusion is that the angles that the transverse line forms with the parallel line are falsely estimated (Hering, 1861). It is argued that all acute angles are overestimated. In Figure 1, the transverse lines "turn away" from the parallel lines and, for this reason, they do not appear to form one continuous line. Variations on this theme have been provided by a large number of investigators, many of whom also attempted to provide a neurophysiological basis for the assumed rotation of the arms of acute angles (Blakemore, Carpenter, \& Georgeson, 1970; Burns \& Pritchard, 1971; Chiang, 1968; Ganz, 1966). Despite persistent attempts to show that such a theory is not a sufficient explanation of the distortion (Coren, 1969; Cumming, 1968; Farné, 1970; Judd, 1899; Pressey \& den Heyer, 1968; Pressey \& Dewar, 1970; Pressey \& Sweeney, 1969; 1972a; Restle, 1969), the desire to explain the Poggendorff illusion on the basis of perceptual enlargement of the acute angles persists. For example, Walker (1973) recently has presented an elaborate mathematical theory which is rooted in a concept of "spuriously excited data points." Unfortunately, this theory is simply a different way of reiterating the view that the Poggendorff illusion is due to an apparent rotation of arms of angles. It does not address itself seriously to an explanation of variations of the Poggendorff target that have

This research was supported by the National Research Council of Canada (AO-177). been offered as evidence against an "angle" theory. Several of these variations are shown in Figure 2. Figures $2 \mathrm{~A}, 2 \mathrm{~B}$, and $2 \mathrm{C}$ illustrate the fact that distortions can occur in the classical direction without the presence of either acute or obtuse angles, and Figures 2D, 2E, 2F, and $2 \mathrm{G}$ illustrate marked reversals of the illusion. The "angle" theories simply cannot explain these effects.

A second explanation of the Poggendorff illusion involves the idea that the configuration involves two-dimensional cues to depth or distance and that the brain processes the target as if it were a threedimensional pattern. Such processing produces a perception of noncollinearity of the oblique lines. This approach was suggested as early as 1898 by Filehne; it was reiterated by Green and Hoyle in 1964 and made explicit by Gillam in 1971. Although this theory seems capable of explaining some of the classical facts about the illusion (Rock, 1975), the reversals of illusion illustrated in Figure 2 present difficulties for this theory also.

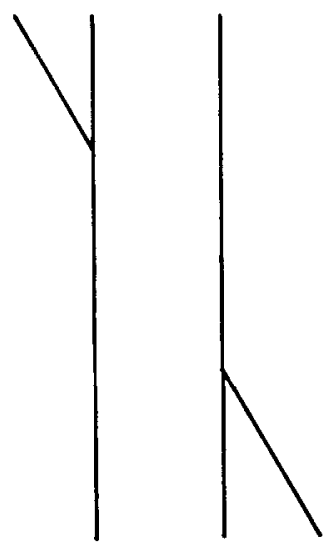

Figure 1. The Poggendorff configuration. 


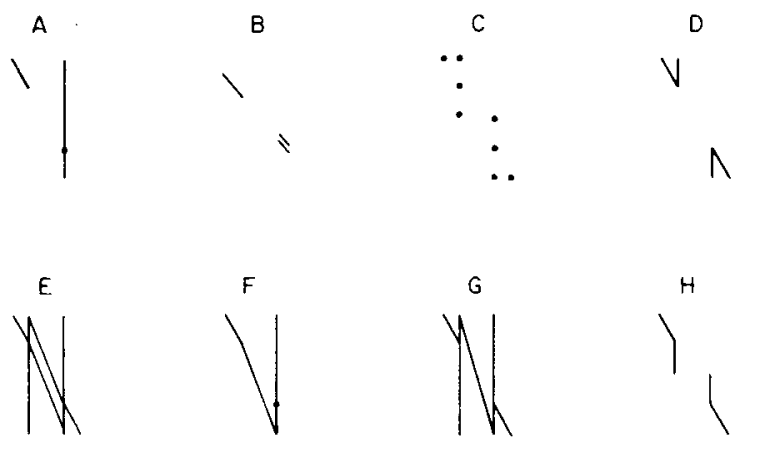

Figure 2. Modifications of the Poggendorff configuration.

A third explanation of the Poggendorff effect reduces it to the classical Müller-Lyer configuration (Pressey, 1971; Pressey \& Sweeney, 1972b). Initially, it was argued that, in a simplified target such as the one shown in Figure $2 \mathrm{~A}$, the observer projects not only the true oblique line onto the far parallel line but also a series of obliques, as shown in Figure 3. In addition, it was argued that, because of the attentive field (see Pressey \& Sweeney, 1972b), the shorter obliques are weighted more heavily than the longer obliques, with the result that the standard magnitude assimilates and appears shorter than it really is. Thus, when the observer chooses a projection from this series, he chooses a line that is shorter than the standard, with the result that the oblique line appears to point above the point of objective continuation.

Although this explanation appears to focus quite properly on the apparent distance between the inner tips of the oblique lines, it is not articulated adequately. In its present form it is contradicted by the target shown in Figure 4 because, in this case, the oblique line can be projected unequivocally only on one spot (the dot), with the result that no illusion should be exhibited. Yet, a powerful illusion does exist (Weintraub \& Krantz, 1971).

In view of this kind of consideration, the theory has been restricted to the statement that "there is a direct relationship between direction and amount of change of the standard magnitude and the direction and amount of Poggendorff illusion" (Pressey \& Sweeney, 1972b, p. 437). The standard magnitude refers to the distance between the points of intersection of the oblique lines with the vertical lines. The argument thus is that the Poggendorff illusion is a compound Müller-Lyer illusion, in which both shrinkage and expansion of the standard magnitude occur simultaneously. When shrinkage is dominant, the distortion is in the classical direction but when expansion is dominant, the illusion is in a reversed direction. Figures $2 \mathrm{D}$ and $2 \mathrm{H}$ exemplify this relationship. The acute angles in Figure 2D produce an outgoing Müller-Lyer illusion, and the obtuse angles in Figure $2 \mathrm{H}$ produce an ingoing Müller-Lyer illusion. But when the two angles are combined, the shrinkage effect is dominant because of the attentive field (see Pressey, Butchard, \& Scrivner, 1971; Pressey \& Sweeney, 1972b). The dominance of the shrinkage effect is shown clearly in Figures 5A and 5B, in which the distance between the dots on the left appears shorter than the distance between the dots on the right.

A systematic application of this restricted explanation of the Poggendorff illusion to the targets shown

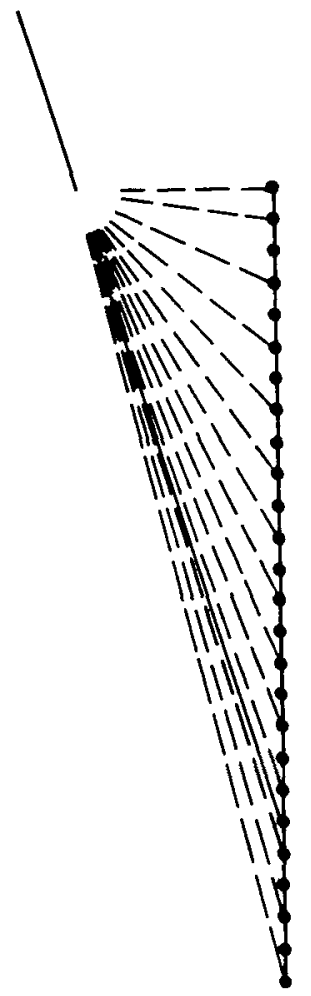

Figure 3. Hypothetical processes involved in the projection of an oblique line onto a vertical line.

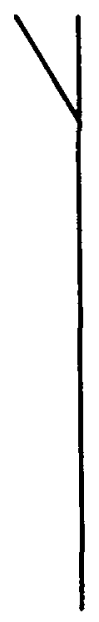

Figure 4. A variation of the Poggendorff target. 
$A$

B

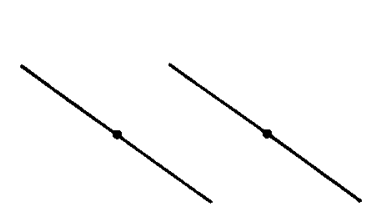

$$
\mid
$$

Figure 5. The distance between the dots in $A$ appears shorter than the distance between the dots in B (after Judd, 1899).

in Figure 2 will reveal that the theory can explain most of the configurations. In every case of a positive illusion, the ingoing form of the Müller-Lyer illusion dominates (according to the attentive field postulate of assimilation theory) and, in every case of a reversal of illusion, the standard magnitude could be viewed as a case in which longer magnitudes are accentuated. According to the generalization, these longer magnitudes should produce a reversal of illusion.

Although the preceding analysis of the Poggendorff illusion appears promising, it is still at the level of a correlational statement and, as such, requires stronger evidence to establish that the correlation is not a fortuitous one. The present study was designed to obtain such evidence.

\section{EXPERIMENT I}

One variable that has not been investigated systematically in the Poggendorff configuration is length of the transverse arm. However, in view of the above arguments and in view of some results obtained by Smith and Sowton in 1907, an assessment of the role of this variable seemed appropriate. Smith and Sowton found that, if they marked the ends of a horizontal line with two short vertical lines to form a standard and then added another modifying line continuous with it, the standard line elongated. Elongation increased and then decreased as the modifying line increased in length.

If the results by Smith and Sowton are reliable, and if it is true that a positive Poggendorff illusion is caused by a subjective shrinkage of the distance between the oblique arms, then increasing the length of the oblique line should result first in a decrease and then in an increase in the Poggendorff illusion. In other words, we expected the illusion to vary as a $U$ function with an increase in the length of the transverse arm.

\footnotetext{
Method

Subjects. The subjects were a total of 49 men and women from an introductory course at the University of Manitoba. Only those with good vision, with or without glasses, were asked to participate.
}

Design. A 2 by 2 by 8 within-subjects design was employed. The variables were (a) distance between parallel lines $(15$ and $30 \mathrm{~mm})$; (b) angle between the oblique line and the parallel line $\left(25^{\circ}\right.$ and $\left.40^{\circ}\right)$; and (c) length of oblique line $(5,10,15,20,25,45,65$, and $85 \mathrm{~mm}$ ). Two experimenters were employed; one experimenter tested 22 subjects, and the second tested 27 subjects.

Materials. Thirty-two targets were drawn in black ink on $21.5 \times 28.0 \mathrm{~mm}$ sheets of white paper. The width of the lines was about $0.5 \mathrm{~mm}$, and the length of each parallel line was $120 \mathrm{~mm}$. The parallel lines were oriented vertically at the center of each sheet. Only one oblique line was used, and it joined the left parallel line at a distance of $35 \mathrm{~mm}$ from the top of that line. All targets were reproduced by of fset duplicating procedures, and they were presented on a $36.8 \times 39.5 \mathrm{~cm}$ white target holder. The face of the holder was tilted backwards $20^{\circ}$ so that the target was perpendicular to the line of sight during testing. A chinrest was located directly in front of the target so that the distance between the eyes and the target was approximately $41 \mathrm{~cm}$.

Procedure. The subject was told that his task was to place, with a sharp pencil, a small, but well-defined, dot on the right parallel line at the point that the oblique line on the left would intersect it if the oblique line were extended. It was emphasized that hand movements were not to assist in making judgments. A target with a large acute angle was employed to demonstrate the task.

Each target was centered on the holder, and the subject made one judgment on each of the 32 targets. The rate of presentation was subject-paced in the sense that, although the experimenter maintained the same rate between trials, the subject was permitted as much time as he required to make his response.

\section{Results and Discussion}

For each target, the oblique line was extended objectively until it crossed the parallel line on the right. Each subject's response was then scored in relation to this point of objective alignment. If the subject's response was above this point, the illusion was scored as positive, if the produced dot was below the point of objective alignment, this was considered to be a negative illusion. Two dependent variables were employed. In one, the score was the linear distance (in millimeters) between the point of objective alignment and the response. In the second case, angular distortion was employed as the measure of illusion, and this was obtained by an application of the law of cosines. The exact method of, and rationale for, using angular distortion has been provided elsewhere (Pressey \& Sweeney, 1972b). Very similar patterns of results were obtained with the two measures but, since the illusion based on angular deviation is the more interesting one theoretically, angular deviation was the dependent variable that was employed.

The amount of distortion is plotted as a joint function of length of arm and distance between parallel lines in Figure 6A and as a joint function of length of arm and angle in Figure 6B. Trend analysis (Myers, 1969) confirmed that the initial prediction of a $U$ function relating illusion to length of arm was verified, $F(1,48)=9.714, p<.01$. Analysis of variance revealed that distance between parallel lines (A) was not significant but angle (B), length of arm $(C)$, and the $B C$ and the $A B C$ inter- 

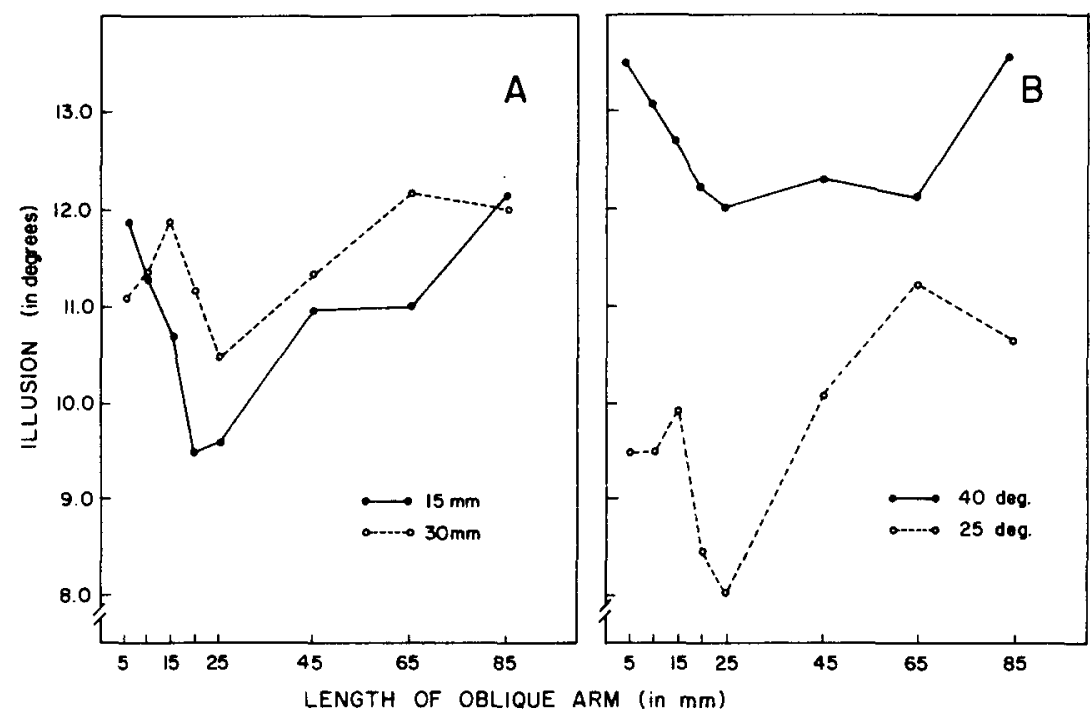

Figure 6. The Poggendorff illusion as a joint function of $(A)$ length of oblique arm and distance between parallel lines and (B) length of oblique arm and angle of oblique arm. actions all produced significant $F$ ratios $(p<.02)$. Thus, the effect of arm length depends upon the angle and the distance between parallel lines, but the present study does not clarify the exact nature of this complex interaction.

\section{EXPERIMENT II}

The results from Experiment 1 , although verifying a prediction from assimilation theory, do not provide unique support for assimilation theory. One of the traditional angle theories might argue that length of oblique arm has an effect on the apparent size of that angle. Specifically, it might be that the apparent enlargement of acute angles decreases at first and then increases as one of the arms increases. A second experiment was designed to determine how the apparent size of an angle varies as one of its arms increases in length.

\section{Method}

Subjects. Sixteen men and women from an introductory course at the University of Manitoba participated. Only those with good vision, with or without glasses, were asked to volunteer.

Materials. Thirty-five millimeter slides were prepared in each of which there were two vertically oriented Poggendorff configurations. When projected, the two targets were separated by a distance of $132 \mathrm{~mm}$. The acute angle of each target was $25^{\circ}$, and the distance between parallel lines was $15 \mathrm{~mm}$. The lengths of oblique arm were $5,10,15,20,25,45,65$, and $85 \mathrm{~mm}$. Each oblique arm joined the left vertical line at a distance of $35 \mathrm{~mm}$ from the top of that line.

Since the method of paired comparisons was employed, each Poggendorff configuration was paired with every other configuration for a total of 28 pairs. In addition, the left-right positioning of the targets was reversed, and this yielded a total of 56 slides.

The slides were presented by means of a random-access selffocusing projector. The targets were presented on a large backprojection screen which was tilted backwards at an angle of $20^{\circ}$. The subject sat directly in front of the screen so that the distance between his eyes and the screen was approximately $41 \mathrm{~cm}$. The screen was perpendicular to the line of sight.

Procedure. The subjects were told that their task was to judge the relative size of acute angles. Two Poggendorff figures, drawn on paper, were presented to the subject and attention was drawn to each of the acute angles. The subject was told that similar figures would be projected on the screen and that he was to select the angle that appeared larger. The subject was to respond verbally with the words "left" if the angle on the left appeared larger and "right" if the angle on the right appeared larger.

Each subject made one judgment on each of 28 targets, which were presented in random order. A different random order was selected for each subject. Targets were visible during the entire period that the subject took to make his response. The intertrial interval was determined by the random-access selector, but no systematic pattern was evident. The left-right positioning of the targets was balanced between subjects.

\section{Results and Discussion}

The first step in the analysis involved a conversion of responses to a common meaning. Then data were analyzed using the Law of Comparative Judgments Case V (Torgerson, 1958). The resulting function which relates apparent size of acute angle to the length of the transverse arm is shown in Figure 7. It is clear that the apparent size of an angle increases, then decreases, as the length of one of its arms increases. Analysis of variance indicated that the effect of length of arm on apparent angle size was statistically significant, $F(7,15)=3.79$, $\mathrm{p}<.05{ }^{\prime}$ and trend analysis showed that the quadratic component of this function was reliable, $F(1,15)=$ $11.138, \mathrm{p}<.01$.

The fact that the apparent size of an angle increases and then decreases with length of transversal contradicts the argument that apparent size of angle causes the Poggendorff illusion, since the Poggendorff illusion decreases and then increases as the length of the transversal increases.

Two other facts that emerge from this study are 


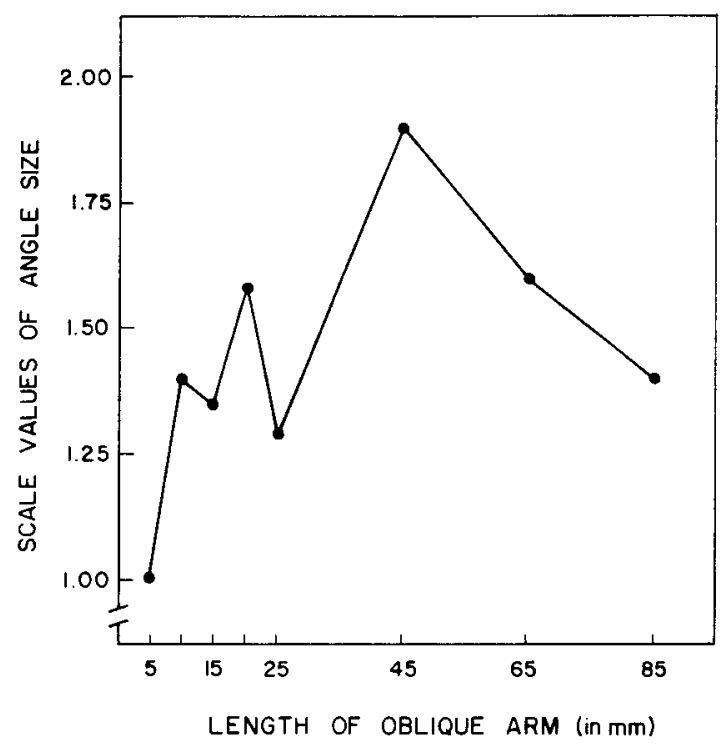

Figure 7. Apparent size of angle in Poggendorf target as a function of length of oblique arm.

also difficult to reconcile with an angle theory. These facts are based on the use of angular deviation as a measure of illusion. First, the illusion increases when the distance between parallel lines increases (Figure 6A). Although the effect was not statistically significant in the present experiment, the direction of difference replicates a previous result reported by Pressey and Sweeney (1972b). But if the Poggendorff distortion is due to subjective rotation of the transversal, it is difficult to understand why variation in the distal parallel line affects the degree of rotation. A second result that is difficult to explain is that distortion is greater when the oblique arm forms a $40^{\circ}$ angle with the parallel than when it forms a $25^{\circ}$ angle. In order to determine the generality of this result, Velinsky's data (1925) on the Poggendorff illusion were converted into angular distortion. His results are presented in Figure 8. These data confirm the results of the present study and also show that the maximum effect occurs at an angle of about $40^{\circ}$.

The fact that maximum distortion is produced by a moderately large angle $\left(40^{\circ}\right)$ is damaging both to physiological and judgmental angle theories. For example, Blakemore et al.(1970) argue that inhibition between orientation detectors causes illusions such as the Poggendorff. But the evidence suggests that the angular deviations that produce optimum interactions among orientation detectors is very much smaller than $40^{\circ}$ (Hubel \& Wiesel, 1968). Thus, superficially at least, the physiological and behavioral data seem incongruous.

Judgmental theories of angular distortion face a similar difficulty. One of the strongest arguments that angle theories make is that distortion produced by the Poggendorff, Hering, and Zöllner targets are all explicable on the same principle. But again, the evidence seems inconsistent since it has been found repeatedly that an angle of between $15^{\circ}$ and $20^{\circ}$ provides the largest Zöllner illusion (Robinson, 1972) and not one of $40^{\circ}$ as would be expected from the data shown in Figure 8.

\section{EXPERIMENT III ${ }^{2}$}

Experiment I showed that the effect of increasing length of transversal was consistent with the view that the Poggendorff illusion is due to apparent shrinkage of the distance between transversals. However, there is an ancient (and obscure) fact that is disturbing for this theory. In 1901, Pierce reported that two observers, when asked to estimate the distance between transversals by a method of adjustment, produced an overestimation of that distance. This result contradicts not only assimilation theory but seems to be incompatible with Judd's illustration shown in Figure 5.

However, the significance of Pierce's (1901) finding is indeterminate because of a major problem associated with any direct attempt to relate apparent length to apparent orientation or displacement in the Poggendorff figure. If a comparison figure is presented simultaneously with (and near to) the Poggendorff figure, the resulting stimulus configuration is different from one in which no comparison stimulus is present. Consider Figure 9, in which the distance between the two dots, $A B$, is

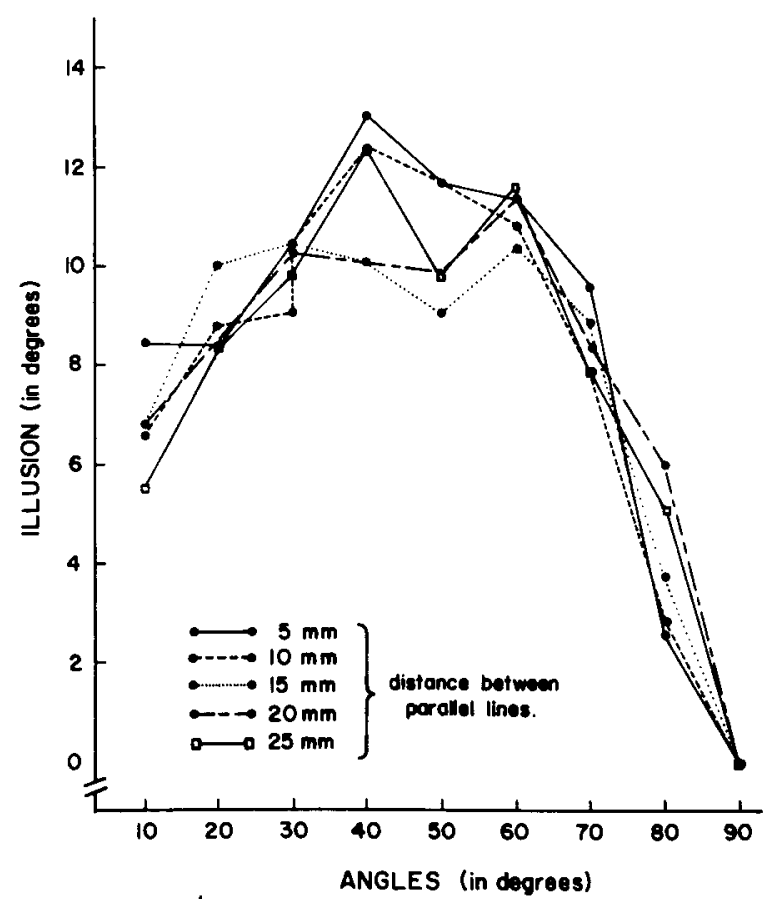

Figure 8. Conversion of Velinsky's (1925) data on Poggendorff illusion into angular deviation. 


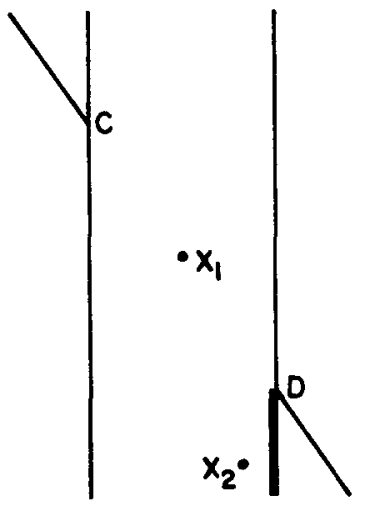

A of this experiment was to obtain measures of the apparent distance between the inner tips of the oblique lines and to relate this measure to the classical Poggendorff effect. The relationship was to be established in two ways. First, measures were to be obtained from targets in which angles of oblique lines varied, and second, the same subjects were to be employed in any one condition in order to calculate correlation coefficients between measures of apparent distance and apparent orientation.

\section{Method}

Subjects. The observers consisted of 190 introductory psychology students enrolled at the University of Manitoba. Only those with good vision, with or without corrective lenses, were asked to participate. Ten subjects were eliminated because they failed to follow instructions.

Materials. Fifteen targets (five Poggendorff, five Poggendorff distance, five control figures) and five response figures were drawn with black ink on $21.5 \times 28.0 \mathrm{~cm}$ sheets of white paper. The Poggendorff configuration consisted of two parallel, vertical lines which were $160 \mathrm{~mm}$ long and $30 \mathrm{~mm}$ apart. The transversal was $\mathbf{4 0} \mathrm{mm}$ long and intersected the left vertical line $40 \mathrm{~mm}$ from its upper tip. The size of the acute angle formed between the transversal and the left vertical line varied between $20^{\circ}$ and $60^{\circ}$ in steps of $10^{\circ}$.

The Poggendorff distance targets were identical to the Poggendorff configurations, except that a black dot was placed on the right vertical line at the point where the transversal, if objectively extended, would intersect the line.

The control figures consisted of two dots. The placement of the dots coincided with the point of intersection of the transversal with the left vertical line and the point where the transversal, if objectively extended, would intersect on the right vertical line.

Identical response figures were used for each pair of Poggendorff distance and control targets. These response figures each contained one dot. The dot's position on the response sheet varied among each of the five pairs of Poggendorff distance and control targets so that the horizontal distance on either side of veridical re' jonses would be roughly equal.

The targets were black contours (approximatley $.5 \mathrm{~mm}$ wide) produced by offset printing on white sheets of paper. The dots on the targets were $2 \mathrm{~mm}$ in diameter. During testing, the targets were centered on the white target holder described in Experiment I.

Design. The experiment used a 5 by 3 mixed design. There were five levels of the between-subject factor (angle) and three levels of the within-subject factor (the task required of the subject).

Thirty-six subjects were randomly assigned to each of the five levels of angle.

Procedure. For the Poggendorff task, the method of production (Pressey, 1974) was used. The subject was told to place a small dot on the right vertical line at the point where the transversal, if extended, would appear to intersect it.

In the Poggendorff distance task, a method of successive production (adapted from Taylor, 1961) was employed. The subject estimated the distance between the point that the transversal intersected the left vertical line and the dot on the right vertical line. The target was then immediately replaced by the response figure. The subject then made his estimate by placing a dot directly to the right of the dot on the response figure so that the distance between the two dots appeared equal to the judged distance.

For the control task, the procedure was identical to the Poggendorff distance task. The subject estimated the distance between the two dots and then made his estimate of the judged distance on the response figure. tortion while keeping the attentive field as nearly alike as possible in the two tasks. Thus, the purpose 
Subjects were tested twice on each of the three tasks. The order in which the tasks were performed was counterbalanced between subjects of the same group. During the testing, each subject was required to view the target for $5 \mathrm{sec}$ before making his response.

\section{Results}

Measurements were made with a straight-edge scale and were accurate to the nearest millimeter. For the Poggendorff illusion, the dependent variable was the distance between the dot that the subject drew and the point at which the transversal intersected the left vertical line. ${ }^{3}$ For the control and the Poggendorff distance tasks, the dependent variable was the distance between the center of the dot on the response sheet and the center of the dot that subject drew.

The scores on the Poggendorff distance task were corrected by subtracting out, for each subject, the score on the control target. The scores on the Poggendorff illusion task were corrected by subtracting out the objective distance between the apex of the angle and the point that the oblique line would intersect the distal line if that oblique line were extended.

The results are shown in Figure 10. As expected, the Poggendorff illusion decreased as the angle increased. However, the results on apparent distance were unexpected, because the distance between the apex of the angle and the distal dot was overestimated. In other words, the distance between the oblique lines in a Poggendorff figure appears longer than an equal distance that is demarcated by two dots. The effect is highly reliable since, for example, at $20^{\circ}, 30$ of the 35 subjects exhibited elongation. The decline in the amount of elongation as the angle of the oblique arm increased was also reliable $[F(4,175)=5.06, p<.01]$.

For each size of angle, Pearson product-moment correlation coefficients were calculated between the Poggendorff illusion scores and the apparent distance scores. The coefficients were $.09,-.14$, $-.01, .18$, and -.27 for the $20^{\circ}, 30^{\circ}, 40^{\circ}, 50^{\circ}$, and $60^{\circ}$ angles, respectively. None was significant at the .05 level of confidence.

\section{EXPERIMENT IV}

It might be argued that the subjective elongation of the distance between the oblique arms found in Experiment III was due to the effect noted by Smith and Sowton in 1907. That is, the oblique arms are extensions of the distance between those arms, and thus should produce subjective expansion of that distance.

In order to provide a control for the role of the arm itself, a target, without an oblique present, was constructed. The target was identical to the control

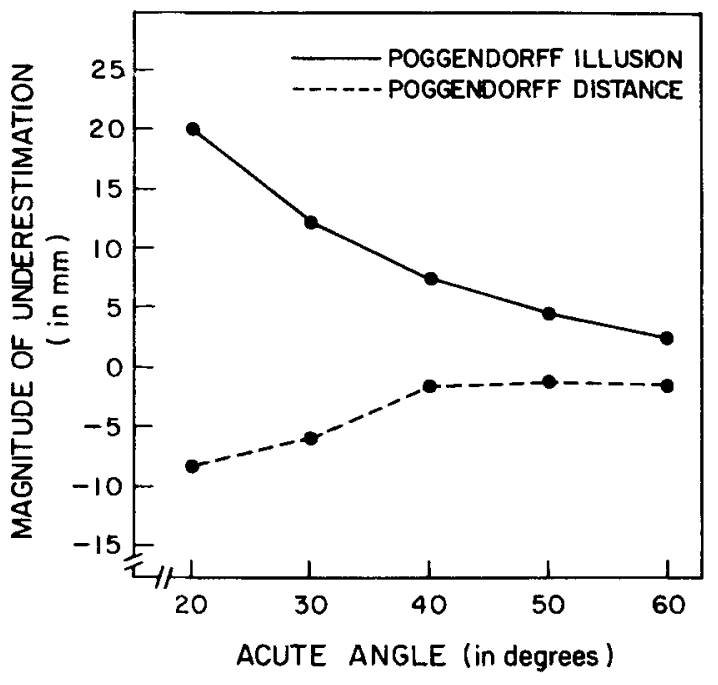

Figure 10. Amount of underestimation of the distance between the oblique lines in the Poggendorff illusion and the Poggendorff distance tasks as a function of angle of oblique arm.

figure (for an angle of $20^{\circ}$ ) used in Experiment III, but with the long vertical lines included. Ten subjects were tested on this new target and on the appropriate control target with the method used in Experiment III.

The results showed that 9 of the 10 subjects exhibited elongation of the standard distance as compared to the control distance. The amount of overestimation was $10.6 \mathrm{~mm}$, which was statistically significant $(t=3.98, p<.01)$. Thus, the argument that the results obtained in Experiment III are due to the elongation effect reported by Smith and Sowton is not tenable.

\section{DISCUSSION}

The results of Experiment III contradict the argument that apparent shrinkage of the distance between the oblique lines causes the Poggendorff effect. When a direct measure of apparent distance is obtained, the illusion appears to be related to phenomenal expansion rather than shrinkage. And, of course, this is precisely the conclusion reached by Pierce in 1901 .

The assimilation theory of the Poggendorff illusion (Pressey, 1971) argues that the classical Poggendorff effect is produced by apparent shrinkage of the distance between the inner tips of the oblique line, and that reversed Poggendorff effects are produced by apparent elongation of that distance. The results presented here clearly indicate that such an argument must be reexamined. Nevertheless, it may be inappropriate to reject entirely the explanation proposed by assimilation theory. One of the major differences between assimilation theory and other explanations of the Poggendorff 


\section{REFERENCES}

Hintzman, D. L., Carre, F. A., Eskridge, V. L., Owens, A. M., Shaff, S. S., \& Sparks, M. E. "Stroop" effect: Input or output phenomenon? Journal of Experimental Psychology, $1972,95,458-459$.

Hock, H. S., \& Egeth, H. Verbal interference with encoding in a perceptual classification task. Journal of Experimental Psychology, 1970, 83, 299-303.

KEELE, S. W. Attention demands of memory retrieval. Journal of Experimental Psychology, 1972, 93, 245-248.

KIEIN, G. S. Semantic power measured through the interference of words with color-naming. American Journal of Psychology, 1964, 77, 576-588.

STRoop, J. R. Studies of interference in serial verbal reactions. Journal of Experimental Psychology, 1935, 18, 643-662.

(Received for publication June 9, 1976; revision accepted September 24, 1976.) 